\title{
Ancillary Diagnostic Tests in Cattle: A Range of Surgical Procedures
}

\section{Jos J Vermunt*}

Adjunct Professor (Cattle Health and Production), College of Public Health, Medical and Veterinary Sciences, James Cook University, Townsville, QLD, Australia

*Corresponding author: Jos J Vermunt, 80 Russell Robertson Drive, Havelock North 4130, New Zealand.

Received date: 29 January, 2022 |

Accepted date: 08 February, 2022 |

Published date: 28 March, 2022

Citation: Vermunt JJ. (2022) Ancillary Diagnostic Tests in Cattle: A Range of Surgical Procedures. J Clin Vet Res 2(1): doi https://doi.org/10.54289/JCVR2200101

Copyright: ( 2022 Vermunt JJ. This is an open-access article distributed under the terms of the Creative Commons Attribution License, which permits unrestricted use, distribution, and reproduction in any medium, provided the original author and source are credited.

\section{Summary}

In addition to a detailed clinical examination, certain diseases or disorders in cattle may require ancillary laboratory tests and/or additional diagnostic procedures before a definitive diagnosis is reached. The present paper provides an overview of a range of surgical diagnostic procedures that may be relatively easily undertaken in clinical cattle practice, including exploratory laparotomy, rumenocentesis, abdominocentesis, liver biopsy, pericardiocentesis, pleurocentesis, lung biopsy, CSF tap/spinal tap, renal biopsy, hoof biopsy, needle aspirates, and bone marrow sampling.

Keywords: Clinical examination; diagnosis; ancillary tests; surgical procedures; cattle

Abbreviations: CSF: Cerebrospinal Fluid, EBL: Enzootic Bovine Leukosis

\section{Introduction}

Clinical reasoning and the art of diagnosis are central to the role of the large animal veterinarian in production animal medicine. When treating an individual animal, observing, and interpreting change in its behaviour, body function and production underpin the establishment of a diagnosis. Careful and methodical observation is required to identify and characterise the ways in which an animal deviates from normal; a detailed clinical examination is then needed to interpret the observations into a diagnosis. The diagnosis might involve a condition expressing as frank clinical disease, a condition in which the signs are very mild, or a condition simply involving reduced performance. Once an accurate diagnosis has been made, the options for clinical management of the condition can be examined and evaluated.
Making a diagnosis may be a simple or a complex procedure. Some diseases are easy to recognise clinically, while others may require a more detailed clinical examination of a specific organ system or systems, careful interpretation of key findings, ancillary laboratory tests or additional diagnostic procedures, if an accurate diagnosis is to be made. Examples of complex diagnoses are abdominal catastrophes, subacute rumen acidosis, diffuse liver disease, neurological disease, and trace mineral deficiencies. The present paper gives an overview of a number of surgical procedures, serving as ancillary diagnostic tests, which may be undertaken in cattle in the field by experienced clinicians without too much difficulty. 


\section{Exploratory laparotomy}

During routine clinical examination of a sick animal, abnormalities may be suspected but not obvious in one or more organ systems that are located in the abdominal cavity. A standing, left-side or right-side exploratory laparotomy (celiotomy) is a relatively fast, inexpensive, and very useful diagnostic procedure to examine those systems in more detail [1]. Research indicates that exploratory laparotomy may provide additional information for the diagnosis of $20 \%$ of cases undiagnosed by standard clinical examination and clinical pathology tests [2]. With good technique and proper attention to asepsis, it is a fairly straightforward and safe procedure that may provide a wealth of information and, in surgical conditions of the rumen, abomasum, intestinal tract and uterus, also provides access for treatment. However, before embarking on surgery, it is absolutely essential that one has a sound knowledge and a clear mental image of the character and anatomical position of the various abdominal organs and structures [3]. Also, the accuracy of diagnosis should be maximised before an unnecessary laparotomy is done. The procedure is indicated if no specific diagnosis can be made, but where the condition appears to be associated with the gastrointestinal tract or uterus, especially if:

- the heart rate is greater than 100 beats/min.

- there is gastrointestinal hypomotility, along with absence or marked reduction in faecal passage.

- there is abdominal distension due to pooling of fluid in the abomasum, intestines or caecum.

- there is abdominal pain of longer than 8 hours duration.

- a gas cap(s) or 'pings' are detected on the right side of the abdominal wall [3].

The surgery should be carried out on the standing animal, which facilitates intra-abdominal exploration and manipulation. In the standing animal there is minimal intraabdominal pressure, which reduces the risk of evisceration. The animal should be restrained in a crush, or any other place that provides ample access to the left or right flank, depending on the indication. A head bail is desirable but placing the animal against the front gate of the left bail (for a left-flank approach) or right bail (for a right-flank approach) of a herringbone shed may offer the best restraint that is available.
However, in such situations there is less protection for the surgeon than when using a suitable crush. Most animals need no sedation, particularly when their clinical condition is serious. In the case of fractious animals, xylazine HCL (0.02 to $0.03 \mathrm{mg} / \mathrm{kg}$ live weight) may be administered intravenously to provide mild sedation.

The incision site should be chosen on the basis of the suspected problem. For example, the left paralumbar region is best in a case of traumatic reticuloperitonitis, whilst the right paralumbar fossa is indicated for conditions such as abomasal volvulus, caecal dilation and volvulus, intussusception and intestinal phytobezoar. An area approximately $20 \mathrm{~cm}$ wide and $30 \mathrm{~cm}$ long should be prepared for aseptic surgery and analgesia provided by a line, an inverted ' $\mathrm{L}$ ' or paravertebral block. The clinician's arm (all the way up, including the armpit and shoulder) should be bare, washed and scrubbed thoroughly. Arm-length rectal examination gloves should not be used, as these are too damaging to the delicate intra-abdominal tissues; they also significantly reduce the sense of touch. However, due consideration should be given to wearing surgical gloves, particularly by those clinicians who do not wear protective gloves for claw trimming and other work in cattle practice that causes gross contamination of the hands.

The paracostal incision should be started 5 to $7.5 \mathrm{~cm}$ ventral to the transverse process of the second lumbar vertebrae and proceed ventrally and parallel to the last rib for about $20 \mathrm{~cm}$. Some clinicians choose for a simple straight incision, whilst others prefer to use a muscle-spreading incision in which each layer is cut in the direction of its fibres. For the latter, the 'key-hole' that is created permits adequate access for the surgeon to explore the abdominal cavity, to perform surgical procedures such as rumenotomy and omentopexy, and is easy to close afterwards. The original incision can be extended downwards, or an alternative incision can be made if other surgical procedures that require better or more access are to be carried out.

After opening the abdominal cavity, visible features - such as volume and colour of peritoneal fluid, appearance, and position of the greater momentum with descending duodenum - should be checked out before the abdominal contents are examined. To minimise peritoneal cavity 
contamination, a thorough and systematic exploration should always be carried out before manipulating or incising any abdominal viscus.

In the case of a left flank approach the following features can be palpated routinely: rumen, spleen, reticulum and its associated diaphragmatic area, left margin of the liver, left kidney, viscera within the omental bursa, uterus and bladder. Following this exploration, any indicated surgery may then be carried out. With right-flank laparotomy, the greater omentum and mesoduodenum with the descending duodenum, the abomasum and pyloric area, omasum, visceral surface of the liver, gall bladder, right half of the diaphragm, visceral structures within the omental bursa (small and large intestine, and caecum), left and right kidney, bladder and uterus are all palpable features. If indicated, this examination should then be followed by any specified surgical procedure(s).

Closure of the laparotomy incision should be done in a routine fashion, avoiding any dead space, especially between muscle layers. The surgeon should be mindful that the internal abdominal muscle (transverse abdominus muscle) layer on the cranial aspect of the incision tends to slip forward and therefore may be easily missed, particularly when suturing all muscle layers together in a simple continuous pattern [2]. Routine intra-abdominal medication is rarely indicated but, depending on the procedure, prophylactic antibiotics (e.g., procaine penicillin) should be administered systemically for 3 days.

\section{Rumenocentesis}

With increasing levels of concentrate feeding in many dairy herds worldwide, acute and subacute rumen acidosis are becoming issues of increasing importance [4]. Possibly the most sensitive measure of rumen health and function is assessment of the rumen microflora [5]. Normal rumen liquor (fluid) contains large numbers of protozoa with ciliate and flagellate forms that are highly active. One of the first changes in rumen acidosis (even before the $\mathrm{pH}$ falls significantly) is reduced motility of protozoa; later in the course protozoa can no longer be found [4].

Methods of obtaining samples of rumen fluid from live cows include oral or nasal intubation, and rumenocentesis (rumen paracentesis). Contamination with bicarbonate-rich saliva is often a problem when using oral or nasal intubation. The most accurate procedure for measuring rumen $\mathrm{pH}$ in the field is rumenocentesis, as the obtained rumen fluid sample will be free of saliva contamination. In simple terms, it involves inserting a needle into the main body of the rumen and aspirating a sample of rumen fluid.

Various techniques of rumenocentesis have been reported. However, in the author's experience, the procedure described by Nordlund and Garrett [6] provides the most consistent results. With this particular technique, light sedation is recommended, although not essential. Hobbling the rear legs or securing the near-rear limb is strongly advised. Alternatively, a 'tail jack' (pushing up the tail) should be applied by an assistant during the sampling procedure. At the same time the cow's head should be pulled to the right side, using a halter or a pair of nose grips.

Landmarks for the puncture site are the left side on a horizontal line level with the top of the patella, approximately 15 to $20 \mathrm{~cm}$ posterior to the last rib or 15 to $20 \mathrm{~cm}$ caudoventral to the costochondral junction of the last rib. The ventral sac of the rumen must be clearly identified beneath the body wall before sampling is attempted. A $2 \times 2 \mathrm{~cm}$ area should be clipped and aseptically prepared in a standard fashion. A 14- to 16-gauge x 4-inch needle should initially be inserted through the skin only. Usually, the animal will object most when penetrating the skin. When the animal is settled the needle should be inserted to the hub with a smooth, but firm thrust. With the needle point positioned in the rumen, rumen fluid may be aspirated with a 10 to $20 \mathrm{~mL}$ syringe with an eccentric tip, using very slight suction. The needle may become clogged with food particles; these can be dislodged by forcing a small volume of air or fluid back through the needle. By holding the syringe with the eccentric tip uppermost, most of the collected fluid will be retained in the syringe while clearing the needle with air. When the needle becomes blocked, it is important not to create a negative pressure within the syringe as $\mathrm{CO}_{2}$ will leave the fluid, thereby artificially increasing the $\mathrm{pH}$ of the sample. Typically, 3 to $5 \mathrm{~mL}$ of rumen fluid can be collected without too much difficulty. When a sufficient volume has been obtained, any excess air should be evacuated from the syringe and the $\mathrm{pH}$ measured immediately. 
The technique of rumenocentesis described above may not be completely benign in healthy cattle, due to the risk (albeit small, 1 to $2 \%$ of cows sampled) of subcutaneous abscess formation around the puncture site. The risk of complications is increased if too short a needle is used for the procedure. When carried out correctly, the possibility of peritonitis developing after rumenocentesis is remote [5]. However, in heavily pregnant cows the procedure should be done with great caution because part of the gravid uterus is often positioned between the rumen and the left abdominal wall.

\section{Abdominocentesis}

In conditions where excess free peritoneal fluid exists in the abdominal cavity, it is sometimes possible to obtain such fluid using abdominocentesis (abdominal paracentesis). Normal cattle may yield 1 to $5 \mathrm{~mL}$ of clear, serum-like peritoneal fluid containing mesothelial cells, lymphocytes, neutrophils, a few red blood cells and occasional monocytes and eosinophils [7]. Collection of a sample of peritoneal fluid can be a useful aid in the diagnosis of diseases of the peritoneum and intestines. However, the omentum of the cow is extremely efficient at trapping (localising) infection and inflammatory fluid, and many attempts at paracentesis do not yield a sample. Additionally, abnormal peritoneal fluid may be confined to a small area of the peritoneal cavity, which may be missed during abdominocentesis. Nevertheless, it is not a difficult procedure and may be very informative when a sample is obtained. Grossly abnormal samples can be interpreted cowside, (e.g., if there is urine present or turbid inflammatory fluid that clots), but other samples require laboratory examination.

In mature cattle, the choice of sites for abdominocentesis is a problem because the rumen occupies a large portion of the ventral abdominal wall and avoiding its penetration may prove difficult [8]. A number of sites for abdominocentesis have been described. One commonly used site is located left to the ventral midline, approximately 3 to $4 \mathrm{~cm}$ medial and 5 to $7 \mathrm{~cm}$ cranial to the foramen where the left mammary vein enters the ventral body wall. Another recommended site is 10 $\mathrm{cm}$ cranial and $10 \mathrm{~cm}$ to the right of the umbilicus when standing left to the cow and facing towards the head. Alternatively, a site in the anterior abdomen, 8 to $10 \mathrm{~cm}$ caudal to the xiphoid sternum and 8 to $10 \mathrm{~cm}$ lateral (i.e., either to the left or right) from the midline, can be used. Other sites are on the left or right abdominal wall, just cranial to the attachment of the udder to the ventral body wall and medial to the fold of the flank.

The animal should be restrained by applying a 'tail jack' and the selected site clipped, prepared aseptically, and infiltrated with a $2 \%$ local anaesthetic solution. A small scalpel cut is made through the skin, and a teat cannula pushed carefully and slowly through the abdominal wall, which will twitch when the peritoneum is punctured. With care and caution, a 16-gauge $\times 2$-inch hypodermic needle may also be used, in which case local anaesthetic may not be required. Fluid may drip from the cannula, or it may be necessary to connect a syringe to apply suction. Often it may be mandatory to move the cannula or needle back and forth in a few different directions before fluid is obtained. If the initially chosen site does not yield fluid, the process may be repeated at another site a few centimetres caudally. A dry 'tap' cannot be taken to mean that excess fluid is not present in the abdomen, or that a local site of peritonitis is not present.

Occasionally, the cannula or needle may penetrate the rumen or the abomasum, especially when attempts to collect peritoneal fluid are made through the ventral midline, but neither causes any apparent problem [7]. This is common if sharp hypodermic needles are used for abdominocentesis. Rumen liquor is relatively easy to recognise and abomasal fluid can be identified by its low $\mathrm{pH}$.

Fluid should be collected into EDTA tubes for cytology and protein analysis, and into plain tubes for bacteriology and biochemistry. Protein concentrations can be used to distinguish between transudate and exudate, being significantly higher in the latter. Peritoneal fluid can also be cultured for bacteria and analysed for urea.

\section{Liver biopsy}

Liver biopsy can be informative and useful in confirming the presence of liver disease and, in many cases, for determining its aetiology. In cattle, it is a relatively safe and simple procedure in which a small core of liver tissue is removed from the liver via a percutaneously introduced biopsy needle [9]. Diffuse and zonal lesions, as seen in most toxic, infectious, and metabolic liver diseases, can usually be diagnosed by histological examination of biopsy sections. 
This may also give an indication of the extent of liver damage in the sampled lobe, as well as indicate a cause. However, the main deficiency of the technique lies in the small sample obtained: unless the liver change is diffuse the sample may not be representative. Biopsy samples may also be used to assess total liver concentrations of certain trace minerals, usually copper, selenium, and vitamin B12 (for assessing the cobalt status).

The size of the biopsy sample needed is dictated by the laboratory tests to be carried out. Small cores of tissue suitable for histopathology are easily obtained with Tru-cut biopsy needles that should be 14-gauge and either 4 or 6 inches long. The four-inch needle will be of sufficient length in all but very well-finished beef animals. A custom made, reusable liver biopsy instrument with an internal diameter of 2 to $3 \mathrm{~mm}$ is more commonly used to obtain the larger samples required for biochemistry [9].

The site for taking a liver biopsy is the right thoracic wall in the $11^{\text {th }}$ (second to last) intercostal space, at about the level of a line drawn between the upper margin of the right tuber coxa and the ipsilateral elbow. The area over this site should be clipped or shaved, prepared aseptically, and infiltrated subcutaneously with a local anaesthetic solution. In order to minimise haemorrhage, it is important to choose a biopsy site close to the cranial margin of the $12^{\text {th }}$ rib, because the major dorsal intercostal blood vessels run close to the caudal border of the ribs. A small stab incision should be made, the biopsy instrument inserted and, using firm but controlled pressure, directed towards the animal's opposite elbow joint, i.e., slightly cranially and ventrally. Initially there will be contact with the diaphragm, which produces movement of the needle synchronous with respiration. After penetrating the muscular diaphragm (sometimes recognised as a slight 'popping' sensation), the tip of the instrument will be embedded in, or rests on, the liver. Now the trocar should be removed and the needle gently advanced into the liver in a rotating fashion, with minimal resistance. A characteristic 'grating' sensation is usually felt as the needle cuts through the liver parenchyma. Slight suction (about 2 to $3 \mathrm{~mL}$ ) should be applied to the instrument with a $10 \mathrm{~mL}$ syringe, and the instrument removed while maintaining the vacuum. If necessary, the needle may be withdrawn slightly and re-directed one or two more times to ensure adequate sampling. No sample will be obtained if the liver is shrunken or the approach too caudally. If successful, the sample should be ejected from the needle on to a sterile swab. Any excess blood and fluid should be carefully blotted away from the core of liver tissue, before it is placed in fixative (for histopathology) or a sealable container (for trace mineral analysis). Although not strictly necessary, the skin incision may be sutured or closed with a Michel suture clip.

For trace mineral assays, it is best to check the amount of tissue and transport medium required by the laboratory before taking the biopsy. Also, chemical disinfectants should not be used on the skin because of possible contamination of the liver sample, which may potentially influence the analyses. Complications following liver biopsy are not common, and the procedure has been repeated many times on the same animal without injury. Mortalities associated with clostridial diseases have been reported, but one therapeutic dose of procaine penicillin post-sampling appears to be an effective preventative in unvaccinated animals. Occasionally, a branch of a hepatic or portal vein may be penetrated, resulting in a steady flow of venous blood from the needle when the trocar is withdrawn. If so, the needle should be either advanced further into the liver or slightly withdrawn and then redirected into the liver parenchyma, followed by taking the sample as described before. Laceration and subsequent massive haemorrhage from the liver is a potential problem, but apparently occurs very rarely and only when a haemorrhagic predisposition is present. Peritonitis may follow if a liver abscess containing viable bacteria is encountered. Similarly, biliary peritonitis may result if a large bile duct is perforated. Pneumothorax may occasionally occur as a complication of liver biopsy but, if it happens, it is always unilateral and usually resolves within one or two hours.

\section{Pericardiocentesis}

The procedure of pericardiocentesis is used to remove fluid from the pericardial space. In normal animals, only a small amount of clear to slightly straw-coloured pericardial fluid can be obtained. In the case of traumatic pericarditis, a dark, foul-smelling fluid containing large numbers of white blood cells is commonly obtained. Cattle with lymphosarcoma may shed neoplastic lymphocytes into the pericardial fluid. 
Congestive heart failure can be accompanied by an excess volume of pericardial fluid, which has a normal cytological distribution and protein concentration.

Most cases of pericarditis are diagnosed on auscultation of extraneous sounds over the heart area which are associated with the heartbeat, but not with any particular heart sound [10]. These are commonly described as 'washing machine' sounds and are regenerated by the mixing of gas and fluid. Muffling of the heart sounds is another common finding with pericarditis. Collection of a sample of fluid from the pericardial sac may confirm a presumptive diagnosis of pericarditis.

The intercostal space between the $5^{\text {th }}$ and $6^{\text {th }}$ ribs should be clipped, aseptically prepared and infiltrated with a local anaesthetic solution. Using a 14- to 16-gauge over-the-needle catheter with a minimum length of $15 \mathrm{~cm}$ is the preferred method. Alternatively, a $15 \mathrm{~cm}$ spinal needle of size $17 \mathrm{BWG}$ can be used. The catheter, with syringe attached, should be advanced carefully towards the heart, preferably under the guidance of ultrasound. To confirm that the catheter is indeed in the pericardial sac and not the pleural cavity, approximately $20 \mathrm{~mL}$ of air may be injected through the catheter and the heart auscultated on the opposite side of the cow [10]. Washing machine sounds should now be generated and audible.

Fluid obtained by pericardial paracentesis should be collected into EDTA blood tubes for cytology and protein analysis, and into a separate container or plain blood tube for culture, if indicated.

\section{Pleurocentesis}

The technique of pleurocentesis (thoracocentesis or pleural paracentesis) is of value when presence of fluid in the pleural cavity is suspected. Normal cattle have small volumes of clear to slightly turbid yellowish, non-clotting pleural fluid.

The procedure can be safely carried out, with or without ultrasound guidance, under local anaesthesia with the animal restrained in a crush [11]. In a mature cow, a 12- to 14-gauge pleural catheter or hypodermic needle, 8 to $10 \mathrm{~cm}$ in length, should be carefully inserted into the caudal aspect of the $6^{\text {th }}$ or $7^{\text {th }}$ intercostal space, below the level of the suspected fluid line, which has been determined by percussion or ultrasonography. Immediately upon entering the pleural cavity pleural fluid will usually drain out of the needle; sometimes it may be necessary to apply slight suction with a syringe.

Pleurocentesis can be used to obtain samples for diagnostic purposes, and to withdraw excess pleural fluid. Insertion of a catheter or needle into the pleural space can also be used to infuse antibiotic.

\section{Lung biopsy}

Percutaneous lung biopsy is a potentially hazardous procedure. Therefore, its use should be limited to those cases where a histological diagnosis is essential for management of the case, and when such a diagnosis cannot be obtained using less invasive procedures, such as broncho-alveolar lavage and trans-tracheal aspiration [12]. Lung biopsy is of most value in lung conditions of a generalised nature [11].

An area of skin over the $6^{\text {th }}$ or $7^{\text {th }}$ intercostal space, in the midsection of the thorax, should be clipped, aseptically prepared and anaesthetised. A stab incision should be made over the intercostal space. The skin should then be pulled somewhat caudally (so that on removal of the biopsy instrument the skin incision is not in line with the deeper wound, thus minimising the risk of subsequent pneumothorax) and a Tru-cut biopsy needle pushed through the cutaneous incision and intercostal muscles, and into the parenchyma of the lung. The cutting stylet of the Tru-cut ${ }^{\circledR}$ needle should then be advanced to cut the sample, after which the cover should be pushed over the cutting stylet to retain the biopsy sample, after which the biopsy needle should be withdrawn. Samples obtained by this procedure are small and may not be representative of other portions of the lung.

Potential complications of lung biopsy include lung collapse, pneumothorax, haemothorax, haemoptysis, and dissemination of infection.

\section{CSF tap / Spinal tap}

Cerebrospinal (CSF) fluid analysis is helpful primarily for confirming the presence of an inflammatory lesion involving the leptomeninges (e.g., bacterial meningoencephalitis), and investigating potential compressive lesions of the spinal cord [13]. In cattle, the collection and analysis of CSF may deliver quick and sometimes immediate information to the clinician investigating a disease problem in a living animal. When 
correctly carried out, a CSF tap is a relatively safe procedure in cattle, usually without any harmful consequences.

CSF can be collected from the subarachnoid space at two sites: (i) the atlanto-occipital space (cisternal sample), and (ii) the lumbo-sacral space (lumbar sample) [14]. Ideally, the site should be chosen based on being as close as possible to the suspected lesion. However, the significant advantages of the lumbo-sacral site, such as the need for less restraint, the reduced risk of side effects and ease of access in adult animals, means that CSF samples usually come from that site irrespective of where the lesion is [13]. Furthermore, sedation of the animal is usually not required when collecting CSF samples from the lumbo-sacral site. Finally, in the absence of a focal compressive spinal cord lesion, there will be no significant differences between the composition of cisternal and lumbar CSF samples. Hence, there are few, if any indications for cisternal CSF collection in production animals.

The collection of a lumbar sample is facilitated, especially in calves and recumbent adult cattle, by positioning the animal in sternal recumbency with the hips flexed and the hindlimbs fully extended alongside the animal's abdomen. In adult cattle that are ambulatory, lumbar CSF should be collected in the standing animal, which must be adequately restrained in a cattle crush or head bail. Mild sedation may be necessary, particularly when dealing with fractious or nervous animals. The site for the lumbar CSF collection is the midpoint of the lumbosacral space, which should be identified as the midline depression between the last palpable dorsal lumbar spinal process (L6) and the first palpable sacral dorsal spinal process (S2). Another anatomical marker for this site is midway on a transverse line connecting both ilium wings. For effective sampling identification of these landmarks is essential [13]. The sampling site should be clipped, aseptically prepared, and injected with 1 to $2 \mathrm{~mL}$ of local anaesthetic solution subcutaneously. It is important that strict sterility is maintained throughout the collection procedure. In calves, an 18 - to 20-gauge x 1.5-inch hypodermic needle should be used; these do not become blocked, and CSF will well up as soon as the needle point enters the dorsal subarachnoid space. Internal stylets are not required. In adult cattle, an 18-gauge $\mathrm{x}$ 4-inch spinal needle plus internal stylet should be guided through a 14-gauge x 2-inch disposable hypodermic needle for the proximal part of its length. The spinal needle should be slowly (over a period of 10 seconds) advanced at a right angle to the plane of the vertebral column, or with the hub directed $5^{\circ}$ to $10^{\circ}$ caudally. It is essential to appreciate the changes in tissue resistance as the needle point passes sequentially through the subcutaneous tissue and interarcuate ligament. Increased resistance to the insertion of the needle can be appreciated at a depth of 3 to $6 \mathrm{~cm}$, when the interarcuate ligament is penetrated, and a sudden 'pop' felt as a result of the loss of resistance as the needle point exits the ligamentum flavum and underlying dura mater, after which it enters the extradural space. Failure to appreciate the change in resistance to needle travel may result in needle puncture of the conus medullaris. This may produce an immediate pain response, causing unnecessary discomfort to the animal. Movement of the hindlimbs may dislodge the needle point with the risk of causing local trauma and haemorrhage in the leptomeninges, which may contaminate the CSF sample that is ultimately collected.

Once the needle has penetrated the dorsal subarachnoid space and the stylet is removed, CSF will well up in the needle hub within 2 to 3 seconds. Although a CSF sample can be collected by free flow over a period of 1 to 2 minutes in most cases, it may be more convenient to employ gentle syringesuction over 10 to 20 seconds to collect 1 to $2 \mathrm{~mL}$ of CSF, which is adequate for laboratory analysis. Care should be taken to avoid dislodging the point of the needle from the subarachnoid space when the syringe is attached to the needle hub. Fluid can then be analysed for cellularity, protein content and cell morphology.

\section{Renal biopsy}

As a general rule, because of the relatively small piece of tissue obtained with biopsy instruments, renal biopsies should be used for diffuse processes affecting the kidneys where histopathological evaluation can assist in determining a definitive diagnosis. The procedure is rarely indicated in cattle.

A percutaneous renal biopsy of the left kidney can be carried out blindly or with the aid of ultrasound by moving the kidney as close as possible towards the right paralumbar fossa and holding it in position by rectal palpation [15]. After making a 
stab incision over the area, an assistant then introduces a Trucut biopsy needle through the abdominal wall.

\section{Hoof biopsy}

Although seldom used in clinical cattle practice, hoof (or claw) biopsies may be indicated in research situations where serial changes during the progress of a particular claw disease, such as laminitis, need to be studied over a period of time [16].

The animal should be adequately restrained in a claw trimming crush or on a tipping table. The claws should be cleaned, pared, and washed thoroughly with a povidone iodine solution. Regional intravenous anaesthesia of the lower limb should be provided by injecting 15 to $20 \mathrm{~mL}$ of a local anaesthetic solution into any accessible vein or venous plexus. When using this particular anaesthetic technique, biopsies can be taken from any part of the claw, such as the parietal corium (via the abaxial wall) and sole corium (via the sole surface).

Using a hand or electric drill with a 7 to $8 \mathrm{~mm}$ bit, a hole is made in the claw horn to remove most of the horn, but without puncturing the underlying corium (i.e., leaving a thin layer of horn covering the corium, which can be judged by the pinkish tinged corium that becomes visible through the horn). Now a 3 to $4 \mathrm{~mm}$ disposable biopsy punch should be used to penetrate through the horn and into the corium (parietal laminae/ sole papillae). The punch should be inserted to its full length and then, after 6 to 8 rotations, carefully withdrawn. Following the removal of the biopsy tissue from the biopsy punch the sample should be fixed immediately in $2.5 \%$ glutaraldehyde or $10 \%$ formol saline. The hole should be filled in with sterile gauze, some type of resin or methyl methacrylate compound. Antibiotics should be administered systemically to reduce the risk of infection.

\section{Needle aspirates (needle biopsy samples)}

Needle aspiration biopsy, also known as fine-needle aspiration, is a diagnostic procedure that is frequently used to investigate superficial lumps or masses. A typical example in cattle is the diagnosis of enzootic bovine leukosis (EBL); enlargement of one or more peripheral lymph nodes occurs in 75 to $90 \%$ of EBL cases that develop lymphosarcoma [17]. With this technique, a thin, hollow needle is inserted into the mass to extract cells that, after being stained, can be examined under a microscope. Fine-needle aspiration biopsies are safe, minor surgical procedures, and a wedge biopsy or node extirpation can often be avoided by using a needle aspiration biopsy instead.

The skin covering the mass to be sampled should be clipped and scrubbed with an antiseptic solution. The skin, underlying fat and muscle may be anaesthetised with a local anesthetic solution, although this is often not needed in cattle with superficial masses. After localising and stabilising the mass, using palpation, a hypodermic needle of fine diameter should be passed into the mass. With the needle is place, cells are withdrawn by aspiration, using a syringe, and subsequently spread onto a glass slide. The needle may have to be alternately inserted and slightly withdrawn a few times, as often several passes are needed to obtain enough cells for cytology/histopathology.

Using this procedure, the risk exists that the abnormal cells will be missed, because the biopsy is very small (only a few cells), resulting in a false-negative result. Another risk is that the cells obtained will not allow a definitive diagnosis to be made.

\section{Bone marrow sampling}

Bone marrow examination is very rarely undertaken in cattle practice. Indications for such examination could be a followup to information obtained from a leukogram (e.g., persistent neutropenia), an anaemic patient (e.g., non-regenerative or non-responding anaemia; unexplained thrombocytopenia), or the suspicion of haemopoietic neoplasia.

The preferred site for reliably obtaining a bone marrow sample in adult cattle is the mid-sternum in the ventral midline at the girth. Because the procedure is painful, deep sedation or anesthesia will be required. After routine skin preparation, a bone marrow trocar with stylet should be tapped into the sternum with a hammer. Once in the marrow cavity, the stylet should be removed, and marrow aspirated with a syringe. Alternatively, a large-bore needle can be used if a bone marrow trocar is not available, although such a needle may plug with bone. In calves, the wing of the ilium is another suitable site for bone marrow sampling. The granules of bone marrow should be placed on microscope slides and immediately prepared as thick smears, which should then be 
air dried.

\section{Other diagnostic procedures}

There are several other ancillary diagnostic procedures or tools that can be used in cattle. Examples are bronchoalveolar lavage, trans-tracheal aspiration, diagnostic imaging, including radiology and ultrasonography, and endoscopy. However, none of these techniques, although invasive for some, have a significant surgical component or, with the exception of ultrasound examination, are routinely used in cattle practice, and thus have not been included in this overview.

\section{References}

1. Desrochers A, Anderson DE. (2016) Intestinal surgery. Veterinary Clinics of North America: Food Animal Practice. 32: 645-671.

2. Vermunt J. (1999) Exploratory laparotomy in cattle. NZ Vetscript. 12(7): 6-7.

3. Malmo J, Vermunt JJ, Parkinson TJ. (2019) Exploratory laparotomy (celiotomy). Diseases of cattle in Australasia - A Comprehensive Textbook, 2nd edn. 115-116.

4. Reference Advisory Group on Fermentative Acidosis of Ruminants (RAGFAR) (2007). Ruminal acidosis aetiopathogenesis, prevention and treatment. A review for veterinarians and nutritional professionals. Australian Veterinary Association (ed).

5. Malmo J, Vermunt JJ, Parkinson TJ (2019) Rumen acidosis. Diseases of cattle in Australasia - A Comprehensive Textbook, 2nd edn. 117-127.

6. Nordlund KV, Garrett EF. (1994) Rumenocentesis: a technique for collecting rumen fluid for the diagnosis of subacute rumen acidosis in dairy herds. The Bovine Practitioner. 28: 104-107.
7. Radostits OM. (2000) Abdominocentesis. In: Radostits OM, Mayhew IG, Houston DM, Veterinary Clinical Examination and Diagnosis. 17-18.

8. Malmo J, Vermunt JJ, Parkinson TJ. (2019) Abdominocentesis or abdominal paracentesis. Diseases of cattle in Australasia - A Comprehensive Textbook, 2nd edn. 114-115.

9. Vermunt J. (2005) Percutaneous liver biopsy procedure in cattle. UK-Vet Livestock 10(3): 31-35.

10. Vermunt JJ, Malmo J, Parkinson TJ. (2019) Pericardiocentesis. Diseases of Cattle in Australasia - A Comprehensive Textbook, $2^{\text {nd }}$ Edn. 306-307.

11. Vermunt JJ, Malmo J, Parkinson TJ. (2019) Pleurocentesis/Lung biopsy. Diseases of Cattle in Australasia - A Comprehensive Textbook, $2^{\text {nd }}$ Edn. 261.

12. Anderson DE, St Jean G. (2008) Surgical procedures of the thorax. Veterinary Clinics of North America: Food Animal Practice. 24: 501-510.

13. Laven R, Vermunt JJ, Malmo J. (2019) Cerebrospinal fluid. Diseases of Cattle in Australasia - A Comprehensive Textbook, $2^{\text {nd }}$ Edn. 430-431.

14. Fecteau G. (2017) Neurologic examination of the ruminant. Veterinary Clinics of North America: Food Animal Practice. 33(1): 1-8.

15. Osborne CA, Fahning ML, Schultz RH. (1968) Percutaneous renal biopsy in the cow and horse. Journal of the American Veterinary Medical Association. 153: 563-570.

16. Singh SS, Ward WR, Murray RD. (1993) Technique of hoof biopsy in cattle. Veterinary Record. 133(8): 190191.

17. Vermunt JJ, Malmo J, Parkinson TJ. (2019) Enzootic bovine leukosis. Diseases of Cattle in Australasia - A Comprehensive Textbook, $2^{\text {nd }}$ Edn. 323-326. 